\title{
A clinical scoring system to predict the need for extensive resuscitation at birth in very low birth weight infants
}

\author{
Juyoung Lee ${ }^{1}$ and Jung Hyun Lee $2^{2^{*}}$
}

\begin{abstract}
Background: To analyze the risk factors for extensive cardiopulmonary resuscitation in the delivery room and develop a prediction model for outcomes in very low birth weight (VLBW) infants.

Methods: The sample was 5298 VLBW infants registered in the Korean neonatal network database from 2013 to 2015. Univariate and multivariate analyses were used to analyze the risk factors for extensive resuscitation. In addition, a multivariable model predicting extensive resuscitation in VLBW infants was developed.

Results: Univariate regression analysis of antenatal factors showed that lower gestational age, lower birth weight, birth weight less than third percentile, male sex, maternal hypertension, abnormal amniotic fluid volume, no antenatal steroid use, outborn, and chorioamnionitis were associated with extensive resuscitation at birth. Lower gestational age (25 to 27 gestational weeks, odds ratio [OR] and 95\% confidence interval [Cl]: 3.003 [1.977-4.562]; less than 25 gestational weeks, OR and 95\% Cl: 4.921 [2.926-8.276]), birth weight less than $1000 \mathrm{~g}$ (OR and 95\% Cl: 1.509 [1.013-2.246]), male sex (OR and 95\% Cl: 1.329 [1.002-1.761]), oligohydramnios (OR and 95\% Cl: 1.820 [1.286-2.575]), polyhydramnios (OR and 95\% Cl: 6.203 [3.185-12.081]), and no antenatal steroid use (OR and 95\% Cl: 2.164 [1.549-3.023]) were associated on multivariate regression analysis. The final prediction model for extensive resuscitation included gestational age, amniotic fluid, and antenatal steroid use. It presented a sensitivity of 0.795 and specificity of 0.575 in predicting extensive resuscitation at birth, corresponding to a score cut-off of 2. The area under the receiver operating characteristic curve was 0.738 .
\end{abstract}

Conclusions: Lower gestational age, abnormal amniotic fluid volume, and no use of antenatal steroid in VLBW infants are important predictors of extensive resuscitation in the delivery room.

Keywords: Neonate, Prediction model, Resuscitation, Very low birth weight

\section{Background}

Most newborn infants make the transition from intrauterine to extrauterine life without difficulty. About 10\% need some assistance, and fewer than $1 \%$ require cardiac compression or medication in the delivery room [1]. However, among very low birth weight (VLBW) infants, approximately $90 \%$ need some kind of resuscitation and 4-10\% require cardiac compression or medication [2-5].

The 2015 American Heart Association Guidelines Update for Cardiopulmonary Resuscitation and Emergency Cardiovascular Care recommend that every birth be

\footnotetext{
* Correspondence: ljhped@catholic.ac.kr

${ }^{2}$ Department of Pediatrics, St. Vincent's Hospital, College of Medicine, The Catholic University of Korea, Jungbu-daero 93, Paldal-gu, Suwon-si, Gyeonggi-do, Republic of Korea

Full list of author information is available at the end of the article
}

attended by at least one person, and that additional personnel with full resuscitation skills should be immediately available for infants with significant perinatal risk factors that increase the likelihood of needing resuscitation [1]. Since most VLBW infants need positive pressure ventilation, two individuals usually attend these deliveries. In addition, when using extensive resuscitation, such as cardiac compression and epinephrine, at least three well-trained personnel, and needed resuscitation equipment and supplies are required.

Medical resources differ between countries and hospitals, as well as at different times of day and days of the week. Although the individual team members may have mastered the skills to resuscitate a newborn, they will not be able to use their skills optimally unless they work

(C) The Author(s). 2019 Open Access This article is distributed under the terms of the Creative Commons Attribution 4.0 International License (http://creativecommons.org/licenses/by/4.0/), which permits unrestricted use, distribution, and 
together as a team. Therefore, it is useful to be able to predict the need for resuscitation earlier than immediately prior to delivery, in order to save medical resources, especially in hospitals where they may be limited. Thus, our goal for this study was to establish a clinical prediction model, and to identify the antenatal risk factors associated with requiring extensive resuscitation in VLBW infants.

\section{Methods}

\section{Study population}

The Korean neonatal network (KNN) database is a national cohort registry of VLBW infants $(<1500 \mathrm{~g})$ born in, or transferred within 28 days of birth to, one of the 66 neonatal intensive care units (NICUs) participating in the KNN. The database includes prospectively collected maternal data recorded at the time of birth, treatment process, and infant outcome data collected from birth until death, transfer, discharge, or 365 days after birth. Each KNN hospital's institutional review board approved data collection for the KNN.

The present study included VLBW infants registered in the KNN database from 2013 to 2015. A VLBW infant who is born and admitted into NICU participating KNN or born in another hospital but transferred to the NICU of the KNN hospital within 28 days of birth was included. Infants with no record of resuscitation, premature rupture of membrane, amniotic fluid, or antenatal steroid use were excluded from analyses.

\section{Definitions of predictor and risk variables}

VLBW was defined as birth weight $<1500 \mathrm{~g}$. Birth weight $<10$ th or $<3$ rd percentile was determined based on sex-specific growth charts [6]. Maternal diabetes was based on diagnosis of gestational diabetes or overt diabetes during pregnancy. Maternal hypertension was based on any maternal diagnosis of pregnancy-induced hypertension or chronic hypertension in pregnancy. Oligohydramnios was defined as amniotic fluid index $<5$. Polyhydramnios was defined as amniotic fluid index $>24$. Antenatal steroid use was defined as any corticosteroid given to the mother during pregnancy to accelerate fetal lung maturity. Complete antenatal steroid status was based on two doses of betamethasone given at a 24-h interval, or four doses of dexamethasone at a 12-h interval, within 7 days before delivery; other administrations were defined as incomplete. Outborn was defined as born at another hospital and transferred to a hospital participating in the KNN. Chorioamnionitis was defined as the presence of acute inflammatory change in the amnion, chorion-decidua, umbilical cord, or chorionic plate based on histologic examination by a pathologist. Extensive resuscitation was defined as administration of chest compression, with or without administration of epinephrine, at birth in the delivery room.

\section{Statistical methods}

Descriptive analyses were performed using Chi-square $\left(x^{2}\right)$ or Fisher exact probability test for categorical variables, and independent $\mathrm{t}$-test for continuous variables. To assess the association between extensive resuscitation and antenatal factors, logistic regression was performed.

To develop a prediction model based on available antenatal data, the data were randomly split into training (70\%) and validation (30\%) sets by statistical package. The data sets were comparable (data not shown). Using the training data set, a multivariable logistic regression model was constructed with extensive resuscitation as the outcome. Variables evaluated for inclusion in the prediction model were limited to those that could be measured before birth: maternal age, diabetes, hypertension, premature rupture of membrane, amniotic fluid volume, gestational age, and use of antenatal steroid. The final model was determined using backward elimination in which significant predictors remained in the model. A weighted scoring system was created using the square root of odds ratios (ORs) in the final model to the nearest integer. Receiver operator curve (ROC) analysis was used to determine the optimum cut-off score to predict extensive resuscitation; this was then applied in the validation set. Statistical analyses were conducted using SAS Version 9.4 (SAS Institute, Cary, NC) and a $P$ value $<0.05$ was considered statistically significant.

\section{Results}

The study sample was 5298 VLBW infants (Fig. 1). A total of 5904 VLBW infants were registered in the KNN database during the study period. Among these, 15 infants had no recorded resuscitation, 44 infants were missing data for premature rupture of membrane, 521 infants were missing data for amniotic fluid, and 117 infants were missing data for antenatal steroid use. As some infants were missing more than one data, 606 infants were excluded and therefore, the final sample was 5298 VLBW infants. Extensive resuscitation occurred in 260 (4.9\%) of these cases.

\section{Associations between antenatal factors and extensive resuscitation}

Infants with lower gestational age, lower birth weight, lower 1- and 5-min Apgar scores, being outborn, and of male sex were associated with extensive resuscitation. Maternal characteristics of the infants who received extensive resuscitation included: hypertension, abnormal amniotic fluid volume (oligohydramnios or polyhydramnios), histologic chorioamnionitis, and no antenatal steroid use. Birth weight $<10$ th percentile, in vitro fertilization, multiple birth, maternal diabetes, premature rupture of membrane, and Cesarean section were not significantly related to the need for extensive resuscitation (Table 1). 


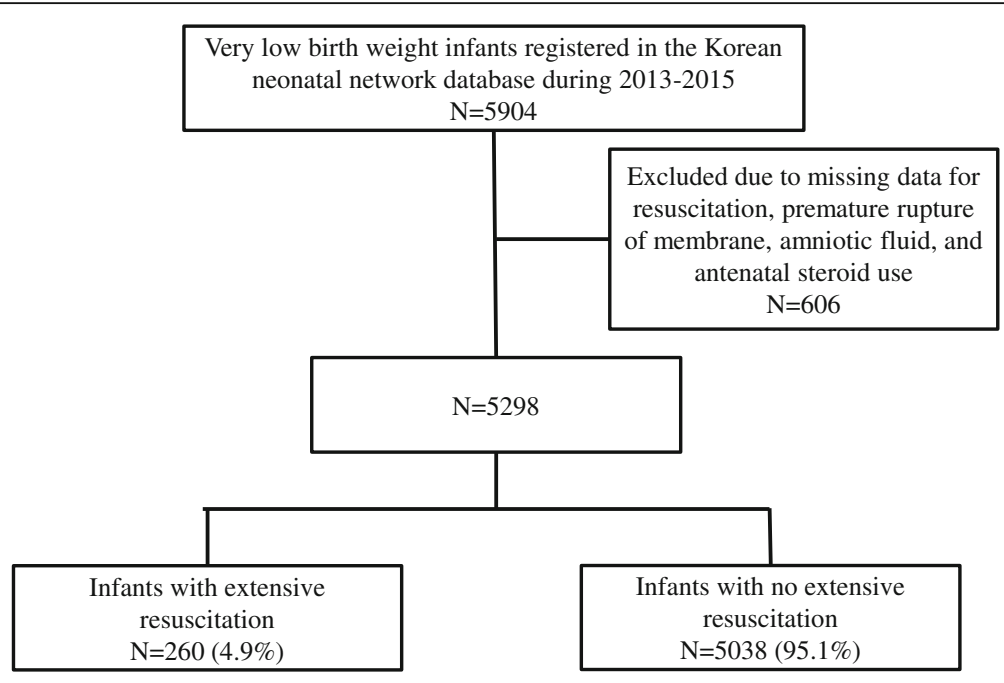

Fig. 1 Flow chart of the study population

Table 1 Maternal and infant characteristics

\begin{tabular}{|c|c|c|c|}
\hline Characteristics & No extensive resuscitation $(n=5038)$ & Extensive resuscitation $(n=260)$ & $P$ value \\
\hline Gestational age (weeks) & $28.96 \pm 2.97$ & $26.53 \pm 2.52$ & $<.001$ \\
\hline Birth weight (g) & $1087 \pm 281$ & $865 \pm 290$ & $<.001$ \\
\hline Birth weight $<10$ th percentile ${ }^{a}$ & 1097 (21.9) & $45(17.6)$ & 0.111 \\
\hline Birth weight $<3 r d$ percentile ${ }^{a}$ & $529(10.5)$ & $16(6.3)$ & 0.029 \\
\hline Male & $2520(50.0)$ & $150(57.7)$ & 0.016 \\
\hline Maternal age & $32.78 \pm 4.18$ & $32.93 \pm 4.20$ & 0.572 \\
\hline In vitro fertilization & $1104(21.9)$ & $46(17.7)$ & 0.107 \\
\hline Multiple births & $1776(35.3)$ & $80(30.8)$ & 0.140 \\
\hline Maternal diabetes & $411(8.2)$ & $17(6.5)$ & 0.350 \\
\hline Maternal hypertension & $1076(21.4)$ & $36(13.8)$ & 0.004 \\
\hline Amniotic fluid & & & $<.001$ \\
\hline Normal & $4255(84.5)$ & $189(72.7)$ & \\
\hline Oligohydramnios & $702(13.9)$ & $55(21.2)$ & \\
\hline Polyhydramnios & $81(1.6)$ & $16(6.2)$ & \\
\hline Premature rupture of membrane & $1775(35.2)$ & $100(38.5)$ & 0.288 \\
\hline Antenatal steroid & & & $<.001$ \\
\hline None & 1105 (21.9) & $94(36.2)$ & \\
\hline Incomplete & $1584(31.4)$ & $70(26.9)$ & \\
\hline Complete & 2349 (46.6) & $96(36.9)$ & \\
\hline Cesarean section & $3880(77.0)$ & $195(75.0)$ & 0.452 \\
\hline Outborn & $88(1.7)$ & $9(3.5)$ & 0.044 \\
\hline Chorioamnionitis $^{\mathrm{b}}$ & 1408 (33.0) & $100(44.2)$ & $<.001$ \\
\hline \multicolumn{4}{|l|}{ Apgar score } \\
\hline 1-min & $4.75 \pm 1.97$ & $1.66 \pm 1.38$ & $<.001$ \\
\hline $5-\min$ & $6.95 \pm 1.66$ & $3.51 \pm 2.11$ & $<.001$ \\
\hline
\end{tabular}

${ }^{a}$ Could not be calculated for 25 infants ( 20 with no extensive resuscitation, 5 with extensive resuscitation) due to gestational age being out of range for the growth chart used

${ }^{\mathrm{b}}$ Not collected for 804 infants (770 with no extensive resuscitation, 34 with extensive resuscitation) 
Multivariate regression analysis of antenatal factors showed that lower gestational age ( 25 to 27 gestational weeks, OR and 95\% confidence interval (CI): 3.003 [1.977-4.562]; less than 25 gestational weeks, OR and 95\% CI: 4.921 [2.926-8.276]), birth weight less than 1000 (OR and 95\% CI: 1.509 [1.013-2.246]), male sex (OR and 95\% CI: 1.329 [1.002-1.761]), oligohydramnios (OR and 95\% CI: 1.820 [1.286-2.575]), polyhydramnios (OR and 95\% CI: 6.203 [3.185-12.081]), and no antenatal steroid use (OR and 95\% CI: 2.164 [1.549-3.023]) were associated with extensive resuscitation at birth. Birth weight $<3$ rd percentile, maternal hypertension, outborn, and histologic chorioamnionitis were associated with the need of extensive resuscitation on univariate analysis, but not on multivariate analysis (Table 2).

\section{Predictive model development}

We excluded sex, birth weight, and histologic chorioamnionitis, which cannot be clearly determined before labor. The final prediction model for extensive resuscitation included: gestational age, amniotic fluid, and antenatal steroid use. For the predictor variables, ORs were calculated and each variable was assigned a score, with the sums of the scores corresponding to an individual infant's risk of requiring extensive resuscitation (Table 3). ROC analysis was used to determine the optimum cut-off value for the score in order to best predict extensive resuscitation. The highest sensitivity and specificity for the training data were 0.795 and 0.575 , respectively (corresponding to a score cut-off of 2) (Table 4). At a score cut-off of 2 , the positive predictive value (PPV) was 0.089 and negative predictive value (NPV) was 0.982 for the training set. The area under the ROC for the training set was 0.738 (Fig. 2). The validation data set model showed sensitivity 0.760 , specificity 0.574, PPV 0.081, and NPV 0.980 at a score cut-off of 2 (Table 5). The area under the ROC for the validation set was 0.714 .

\section{Discussion}

This is the first study to establish a prediction model for extensive delivery room resuscitation in VLBW infants. The model showed fair predictive accuracy.

Few previous studies have explored predictions of the need for neonatal resuscitation [7-10]. Aziz et al. found that maternal hypertension, maternal infection, multiple pregnancy, and oligohydramnios are independent risk factors for requiring positive pressure ventilation and/or endotracheal intubation. Their study included infants at 23 to 42 weeks' gestational age and $9 \%$ were $<36$ weeks [8]. A study by Afjeh et al. with a sample of infants with mean gestation of 37.4 weeks of whom $23.7 \%$ were preterm showed that low birth weight, meconium-stained fluid, and chorioamnionitis are independent risk factors

Table 2 Regression analyses for antenatal factors predicting extensive resuscitation $(N=5298)$

\begin{tabular}{|c|c|c|c|c|}
\hline \multirow[t]{2}{*}{ Antenatal factor } & \multicolumn{2}{|l|}{ Univariate } & \multicolumn{2}{|l|}{ Multivariate } \\
\hline & OR $(95 \% \mathrm{Cl})$ & $P$ value & OR $(95 \% \mathrm{Cl})$ & $P$ value \\
\hline \multicolumn{5}{|l|}{ Gestational age } \\
\hline$>27$ weeks & 1.0 (Reference) & & 1.0 (Reference) & \\
\hline 25-27 weeks & $3.521(2.600-4.768)$ & $<.001$ & $3.003(1.977-4.562)$ & $<.001$ \\
\hline$<25$ weeks & $7.510(5.366-10.509)$ & $<.001$ & $4.921(2.926-8.276)$ & $<.001$ \\
\hline Birth weight < $1000 \mathrm{~g}$ & $3.490(2.678-4.547)$ & $<.001$ & $1.509(1.013-2.246)$ & 0.043 \\
\hline Birth weight $<3$ rd percentile & $0.568(0.340-0.950)$ & 0.031 & $0.836(0.481-1.453)$ & 0.525 \\
\hline Male & $1.363(1.059-1.753)$ & 0.016 & $1.329(1.002-1.761)$ & 0.048 \\
\hline Maternal hypertension & $0.592(0.412-0.847)$ & 0.004 & $1.047(0.693-1.582)$ & 0.826 \\
\hline \multicolumn{5}{|l|}{ Amniotic fluid } \\
\hline Normal & 1.0 (Reference) & & 1.0 (Reference) & \\
\hline Oligohydramnios & $1.764(1.293-2.407)$ & $<.001$ & $1.820(1.286-2.575)$ & 0.01 \\
\hline Polyhydramnios & $4.447(2.551-7.752)$ & $<.001$ & $6.203(3.185-12.081)$ & $<.001$ \\
\hline \multicolumn{5}{|l|}{ Antenatal steroid } \\
\hline None & $2.082(1.552-2.791)$ & $<.001$ & 2.164 (1.549-3.023) & $<.001$ \\
\hline Incomplete & $1.081(0.789-1.481)$ & 0.626 & $0.989(0.702-1.395)$ & 0.951 \\
\hline Complete & 1.0 (Reference) & & 1.0 (Reference) & \\
\hline Outborn & $2.017(1.004-4.052)$ & 0.049 & $0.903(0.209-3.902)$ & 0.891 \\
\hline Chorioamnionitis & $1.612(1.230-2.112)$ & 0.001 & $1.143(0.851-1.536)$ & 0.374 \\
\hline
\end{tabular}


Table 3 Final model for extensive resuscitation

\begin{tabular}{ll}
\hline Variable & Score assigned \\
\hline Gestational age & 0 \\
$>27$ weeks & 2 \\
$25-27$ weeks & 3 \\
$<25$ weeks & \\
Antenatal steroid & 1 \\
None or incomplete & 0 \\
Complete & \\
Amniotic fluid & 0 \\
Normal & 1 \\
Oligohydramnios & 2 \\
Polyhydramnios & \\
\hline
\end{tabular}

for requiring endotracheal intubation [7]. However, it was unclear whether chorioamnionitis in their study was clinically and/or histologically based. In the current study, histologic chorioamnionitis was associated with extensive resuscitation in univariate analysis, but not in multivariate analysis. De Almeida et al. revealed that positive pressure ventilation in late preterm infants (34 to 36 weeks' gestation) was associated with twin gestation, maternal hypertension, non-vertex presentation, Cesarean section, and lower gestational age [9]. Reis et al. proposed the use of a fuzzy expert system based on 61 risk situations to predict the need for positive pressure ventilation, endotracheal intubation, chest compression, and/or medications in the delivery room. Their sample was $10.2 \%$ preterm and $2.6 \%$ were $<34$ weeks' gestation [10].

Notwithstanding the previously established risk factors for neonatal resuscitation described herein, the goal of the present study was to establish early identification of risk factors in order to anticipate the need for personnel skilled in resuscitation. Thus, we included only those factors available in the KNN database that could be determined before birth. Each predictor variable has assigned a score, and the sums of the scores correspond to the infant's risk of requiring extensive resuscitation. If the clinicians don't have access to some of the variables, that pertinent score should be omitted. To date, there have been no data published to develop a clinical scoring system that can be individually used for the prediction of extensive delivery room resuscitation in VLBW infants.
Among the variables included in our prediction model, antenatal steroid use is the only modifiable risk factor. Several studies have shown that late preterm infants born to women who received antenatal steroid required less resuscitation at birth. A randomized trial showed that the betamethasone group required less resuscitation compared with the placebo group [11]. Even receiving a single dose of betamethasone led to less resuscitation [12]. A few studies have also shown that antenatal steroid is related to decreased extensive resuscitation [3]. In a population-based cohort study, infants who received chest compression and/or administration of epinephrine in the delivery room had received less antenatal steroid exposure compared with infants who did not receive extensive resuscitation, based on univariate analysis [13]. The current study supports the notion that antenatal steroid administration is significantly associated with decreased extensive resuscitation, based on univariate and multivariate regression analyses. Our final prediction model also included dose completion of antenatal steroid. The use of antenatal steroid improves lung function, with treated infants having higher Apgar scores and requiring less extensive resuscitation. Other factors may also contribute. The Brazilian Neonatal Research Network observed that antenatal corticosteroid-treated mothers had more prenatal medical visits compared with untreated mothers [14]. More prenatal care could result in improved pregnancy management, such as preventing preterm labor, thereby contributing to improved neonatal outcomes. In addition, there may have been urgent antenatal conditions (which are not provided in the KNN database) that disallowed enough time for steroid dose completion before delivery; such conditions may have independently affected neonates' clinical status. In regression analysis, only no use of antenatal steroids had significant OR. However, prediction model could not be created when antenatal steroid was categorized as none and incomplete/complete. Additional multivariate regression analysis showed no or incomplete antenatal steroid has association with extensive resuscitation (OR and 95\% CI: 1.54 [1.13-2.11]). Classifying antenatal steroid as none/incomplete and complete enabled us to develop a fair prediction model.

Abnormally decreased amniotic fluid volume may reflect fetal dysfunction that prevents normal urination, or it may represent a placental abnormality severe

Table 4 Estimated extensive resuscitation according to the risk score (training set)

\begin{tabular}{lllll}
\hline Score & Sensitivity & Specificity & Positive predictive value & Negative predictive value \\
\hline 1 & $0.924(0.886-0.962)$ & $0.248(0.233-0.262)$ & $0.061(0.052-0.069)$ & $0.984(0.976-0.992)$ \\
2 & $0.795(0.736-0.853)$ & $0.575(0.558-0.591)$ & $0.089(0.076-0.103)$ & $0.982(0.976-0.987)$ \\
3 & $0.643(0.574-0.712)$ & $0.740(0.726-0.755)$ & $0.115(0.096-0.135)$ & $0.975(0.969-0.981)$ \\
4 or more & $0.297(0.231-0.363)$ & $0.925(0.916-0.934)$ & $0.172(0.131-0.214)$ & $0.962(0.955-0.968)$ \\
\hline
\end{tabular}




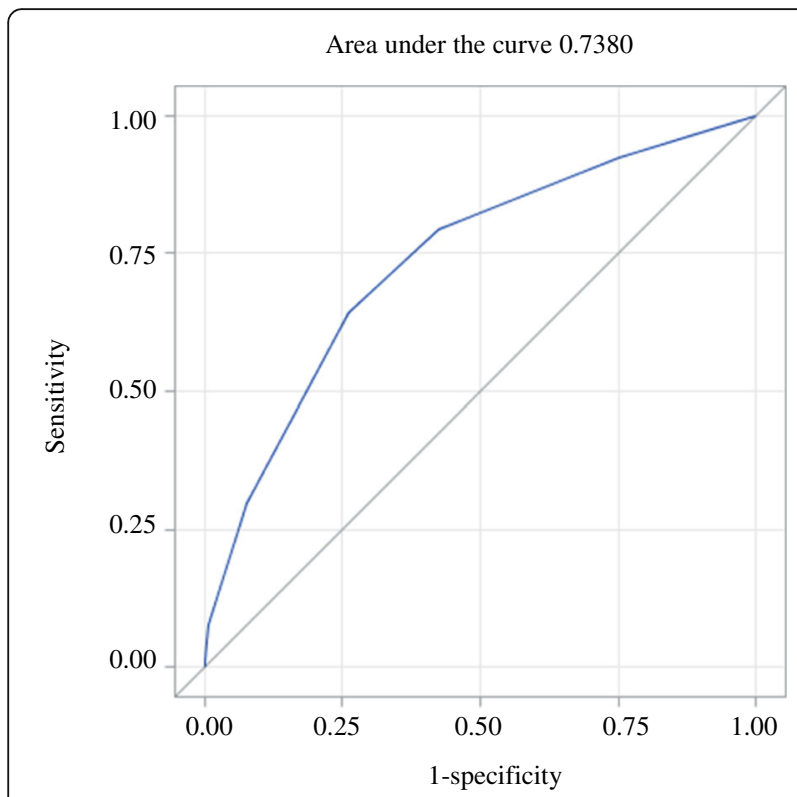

Fig. 2 Receiver operating characteristic curve for the ability of the scoring model to predict extensive resuscitation, training set

enough to impair perfusion [15]. Second-trimester rupture of the fetal membranes may also result in oligohydramnios [16, 17]. Few studies have investigated the correlation between oligohydramnios and resuscitation. Aziz et al. showed that oligohydramnios is a significant risk factor for positive pressure ventilation and/or endotracheal intubation [8]. Our analyses show that oligohydramnios is associated with extensive resuscitation in VLBW infants, and was one of the significant predictors of our model. Our data suggest that infants born to mothers with oligohydramnios who also have other unfavorable maternal and fetal conditions (e.g. fetal or placental insufficiency, premature rupture of membrane) have a worse prognosis at birth, and need more extensive resuscitation in the delivery room, compared with infants born to women with isolated oligohydramnios. This conclusion is consistent with the findings by Zhang et al. [18]

Common underlying causes of polyhydramnios include fetal congenital anomalies (approximately 15\%) and diabetes (15-20\%) [19]. Polyhydramnios is often a component of hydrops fetalis. Data regarding early neonatal complications from idiopathic polyhydramnios are conflicting. Some studies have found higher rate of newborn resuscitation with idiopathic polyhydramnios [20]. However, Panting-Kemp et al. showed that idiopathic polyhydramnios is unassociated with low 5-min Apgar score [21]. Our analyses support the notion that polyhydramnios is associated with extensive resuscitation in VLBW infants.

Our study aim was to develop a prediction model for extensive resuscitation using factors that can be clinically established before delivery. The variables we included were: gestational age, amniotic fluid, and antenatal steroid use. Our data suggest that the delivery by a woman with abnormal amniotic fluid volume, or in whom less antenatal steroid has been administered-despite gestational age $>27$ weeks-should be prepared for extensive delivery room resuscitation. It should be attended by a team composed of at least three well-trained personnel with full resuscitation skills, and that equipment and supplies needed for extensive resuscitation should be prepared.

The present study was limited by only including risk factors available in the KNN database that could be determined before birth. Important variables that would allow assessment of the fetus, such as fetal heart rate, biophysical profile, fetal presentation, placenta abruptio, emergency Cesarean section, were not included in the KNN database. Male sex and extremely low birth weight infant were associated with extensive resuscitation at birth but were not used in the predictive model, because those factors cannot be clearly determined before labor. Another weakness of our predictive model is the low PPV. It results from the very low rate of extensive resuscitation. If a resuscitation team prepare for extensive resuscitation by using our model at a score cut-off of 2 , only 8 out of 100 would be used. It could be safe for VLBW infants but not efficient, especially in hospitals with limited medical resources. Additional data and a larger sample, might improve the model for predicting extensive resuscitation in the delivery room.

The present study outlines factors that significantly increase the need for extensive resuscitation. These findings may be beneficial for developing strategies to anticipate circumstances that require more medical personnel and individuals with advanced neonatal resuscitation skills. Identifying risk factors and anticipating

Table 5 Estimated extensive resuscitation according to the risk score (validation set)

\begin{tabular}{lllll}
\hline Score & Sensitivity & Specificity & Positive predictive value & Negative predictive value \\
\hline 1 & $0.933(0.877-0.990)$ & $0.256(0.234-0.278)$ & $0.059(0.945-0.072)$ & $0.987(0.976-0.998)$ \\
2 & $0.760(0.663-0.857)$ & $0.574(0.549-0.599)$ & $0.081(0.061-0.101)$ & $0.980(0.970-0.989)$ \\
3 & $0.587(0.475-0.698)$ & $0.723(0.701-0.746)$ & $0.095(0.068-0.122)$ & $0.973(0.963-0.982)$ \\
4 or more & $0.293(0.190-0.396)$ & $0.914(0.900-0.928)$ & $0.145(0.089-0.201)$ & $0.963(0.953-0.973)$ \\
\hline
\end{tabular}


adequate levels of resuscitation that may be needed in the delivery room of a VLBW infant may better facilitate adequate preparation and prompt neonatal resuscitation, as well as target limited medical resources for those at the highest risk.

\section{Conclusions}

This study is the first to propose a clinical scoring system to predict extensive delivery room resuscitation in VLBW infants. Lower gestational age, abnormal amniotic fluid volume, and less use of antenatal steroid in VLBW infants are important predictors of extensive resuscitation in the delivery room. However, further studies are required to improve the performance of the prediction model and increase sensitivity of extensive resuscitation in VLBW infants.

\section{Abbreviations}

Cl: Confidence interval; KNN: Korean neonatal network; NICU: Neonatal intensive care unit; NPV: Negative predictive value; OR: Odds ratio; PPV: Positive predictive value; ROC: Receiver operator curve; VLBW: Very low birth weight

\section{Acknowledgements}

Not applicable.

\section{Authors' contribution}

Both authors participated in the study design, analysis, interpretation of data, and discussion. $J \mathrm{~L}$ has involved in drafting the manuscript. JHL participated in review of the manuscript. All authors read and approved the final manuscript. Both authors have agreed to be personally accountable for the author's own contributions and to ensure that questions related to the accuracy or integrity of any part of the work are appropriately investigated, resolved, and the resolution documented in the literature.

\section{Funding}

This work was supported by the research program funded by the Korean Centers for Disease Control and Prevention (2016-ER6307-01\#). Statistical consultation was supported by a grant of the Korea Health Technology R\&D Project through the Korea Health Industry Development Institute (KHIDI), funded by the Ministry of Health \& Welfare, Republic of Korea (grant number: HI14C1062). The funder had no role in the study including design, data collection, analysis, interpretation of data, or writing of the manuscript.

\section{Availability of data and materials}

The data that support the findings of this study are available from KNN network but restrictions apply to the availability of these data, which were used under license for the current study, and so are not publicly available.

\section{Ethics approval and consent to participate}

The study was approved by the institutional review board at Bucheon St. Mary's hospital of the Catholic University of Korea number HC13RNMI0080. Written informed consent to participate in the study was obtained from the parent of the participants. Permission to use the KNN database was obtained from KNN network. Each KNN hospitals' institutional review board approved data collection for the KNN.

\section{Consent for publication}

Not applicable.

\section{Competing interests}

The authors declare that they have no competing interests.

\section{Author details}

'Department of Pediatrics, Bucheon St. Mary's hospital, College of Medicine, The Catholic University of Korea, Seoul, South Korea. ${ }^{2}$ Department of Pediatrics, St. Vincent's Hospital, College of Medicine, The Catholic University of Korea, Jungbu-daero 93, Paldal-gu, Suwon-si, Gyeonggi-do, Republic of Korea.

Received: 12 November 2018 Accepted: 4 June 2019

Published online: 14 June 2019

\section{References}

1. Wyckoff MH, Aziz K, Escobedo MB, Kapadia VS, Kattwinkel J, Perlman JM, Simon WM, Weiner GM, Zaichkin JG. Part 13: neonatal resuscitation: 2015 American Heart Association guidelines update for cardiopulmonary resuscitation and emergency cardiovascular care. Circulation. 2015;132(18 Suppl 2):S543-60.

2. Arnon S, Dolfin T, Reichman B, Regev RH, Lerner-Geva L, Boyko V, Litmanovitz I. Delivery room resuscitation and adverse outcomes among very low birth weight preterm infants. J Perinatol. 2017;37(9): 1010-6.

3. Ballot DE, Agaba F, Cooper PA, Davies VA, Ramdin T, Chirwa L, Rakotsoane $D$, Madzudzo $L$. A review of delivery room resuscitation in very low birth weight infants in a middle income country. Matern Health Neonatol Perinatol. 2017:3:9.

4. Cho SJ, Shin J, Namgung R. Initial resuscitation at delivery and short term neonatal outcomes in very-low-birth-weight infants. J Korean Med Sci. 2015; 30(Suppl 1):S45-51.

5. Finer NN, Horbar JD, Carpenter JH. Cardiopulmonary resuscitation in the very low birth weight infant: the Vermont Oxford Network experience. Pediatrics. 1999;104(3 Pt 1):428-34.

6. Fenton TR, Kim JH. A systematic review and meta-analysis to revise the Fenton growth chart for preterm infants. BMC Pediatr. 2013;13:59.

7. Afjeh SA, Sabzehei MK, Esmaili F. Neonatal resuscitation in the delivery room from a tertiary level hospital: risk factors and outcome. Iran J Pediatr. 2013;23(6):675-80.

8. Aziz K, Chadwick M, Baker M, Andrews W. Ante- and intra-partum factors that predict increased need for neonatal resuscitation. Resuscitation. 2008;79(3):444-52.

9. de Almeida MF, Guinsburg R, da Costa JO, Anchieta LM, Freire LM, Junior DC. Resuscitative procedures at birth in late preterm infants. J Perinatol. 2007;27(12):761-5

10. Reis MA, Ortega NR, Silveira PS. Fuzzy expert system in the prediction of neonatal resuscitation. Braz J Med Bio Res. 2004;37(5):755-64.

11. Gyamfi-Bannerman C, Thom EA, Blackwell SC, Tita AT, Reddy UM, Saade GR, Rouse DJ, McKenna DS, Clark EA, Thorp JM Jr, et al. Antenatal betamethasone for women at risk for late preterm delivery. $N$ Engl I Med. 2016;374(14):1311-20.

12. Balci O, Ozdemir S, Mahmoud AS, Acar A, Colakoglu MC. The effect of antenatal steroids on fetal lung maturation between the 34th and 36th week of pregnancy. Gynecol Obstet Investig. 2010;70(2):95-9.

13. Handley SC, Sun Y, Wyckoff MH, Lee HC. Outcomes of extremely preterm infants after delivery room cardiopulmonary resuscitation in a populationbased cohort. J Perinatol. 2015;35(5):379-83.

14. Network BNR. Antenatal corticosteroid use and clinical evolution of preterm newborn infants. J Pediatr. 2004;80(4):277-84.

15. Bronshtein M, Blumenfeld Z. First- and early second-trimester oligohydramnios-a predictor of poor fetal outcome except in iatrogenic oligohydramnios post chorionic villus biopsy. Ultrasound Cbstet Gynecol. 1991;1(4):245-9.

16. Ulkumen BA, Pala HG, Baytur YB, Koyuncu FM. Outcomes and management strategies in pregnancies with early onset oligohydramnios. Clin Exp Obstet Gynecol. 2015;42(3):355-7.

17. Jabeen S, Shafqat T, Ahmad S. Oligohydramnios causes and pregnancy outcome - third trimester versus second trimester and signficance of amniotic fluid index (AFI). J Postgrad Med Inst. 1997;11(2):182-5.

18. Zhang J, Troendle J, Meikle S, Klebanoff MA, Rayburn WF. Isolated oligohydramnios is not associated with adverse perinatal outcomes. BJOG. 2004;111(3):220-5.

19. Harman CR. Amniotic fluid abnormalities. Semin Perinatol. 2008;32(4): 288-94. 
20. Karahanoglu E, Ozdemirci S, Esinler D, Fadiloglu E, Asilturk S, Kasapoglu T, Yalvac ES, Kandemir NO. Intrapartum, postpartum characteristics and early neonatal outcomes of idiopathic polyhydramnios. J Obstet Gynaecol. 2016;36(6):710-4.

21. Panting-Kemp A, Nguyen T, Chang E, Quillen E, Castro L. Idiopathic polyhydramnios and perinatal outcome. Am J Obstet Gynecol. 1999;181(5 Pt 1):1079-82.

\section{Publisher's Note}

Springer Nature remains neutral with regard to jurisdictional claims in published maps and institutional affiliations.

Ready to submit your research? Choose BMC and benefit from:

- fast, convenient online submission

- thorough peer review by experienced researchers in your field

- rapid publication on acceptance

- support for research data, including large and complex data types

- gold Open Access which fosters wider collaboration and increased citations

- maximum visibility for your research: over $100 \mathrm{M}$ website views per year

At $\mathrm{BMC}$, research is always in progress. 